\title{
Environmentally Smart Contracts for Artists Using Non-Fungible Tokens
}

\author{
Dan Weijers \\ Philosophy Programme \\ University of Waikato \\ Hamilton, New Zealand \\ dan.weijers@waikato.ac.nz
}

\author{
H. Joseph Turton \\ Philosophy Programme \\ University of Waikato \\ Hamilton, New Zealand \\ hjturton@googlemail.com
}

\begin{abstract}
We propose Environmentally Smart Contracts, a new kind of smart contract for non-fungible tokens to solve the prudential-moral dilemma facing digital artists. The current proof-of-work-dominated non-fungible token environment requires artists to trade off the prudential benefits and the environmental costs of selling their art on blockchains. By fully correcting for environmental externalities, Environmentally Smart Contracts will allow artists to reap the sales benefits of non-fungible tokens without contributing to environmental degradation. Concrete steps to encourage the development of Environmentally Smart Contracts are discussed.
\end{abstract}

Keywords—non-fungible token, digital art, environment, blockchain, environmentally smart contracts

\section{INTRODUCTION}

US $\$ 69,000,000$ for digital art? Artists around the world, from seasoned professionals to creative neophytes turned their heads and dropped their jaws when they heard about the first sale of digital art at a major auction house. EverydaysThe First 5000 Days, a digital collage of 5000 digital pictures by Beeple, shocked the art world by selling for just over US\$69 million [1]. Many observers, and even Beeple himself, furrowed their brows - why would someone want to pay so much for a digital picture that can easily be copied [2]? Sure, it's supported by fancy blockchain technology, but that doesn't make the digital picture any more real. At the same time, others began wondering how they could cash in on this digital art revolution [3]. These soon-to-be-rich artists quickly searched online for how to become a part of the blockchain-supported art movement. But their fervour for profit was quickly doused by articles and videos about the huge environmental harms caused by blockchain-supported art [4]. As socially conscious ethical antagonists, they couldn't possibly support something that is so clearly bad for the environment. But as human beings with needs for food, shelter, and financial validation of their creativity, they really wanted to find a way to sell their art digitally. What should they do?

In this paper we provide both concrete but non-ideal steps artists can take now and an ideal multi-party solution that artists and others could support and increase the viability of in various ways. The ideal solution lays out a way for artists to get all of the benefits of selling their art via blockchain technology without perpetuating the environmental problems currently plaguing the industry. First, we explain the relevant technologies and the environmental problem. Second, we discuss the dilemma that the opportunity of selling via blockchain technology poses to artists. Third, we detail some of the steps that artists could take right now to partially resolve the dilemma and support our ideal solution to it. To achieve this, we discuss some innovative uses of Smart Contracts that could be adapted to create a system that dissolves the dilemma for artists, allowing them to sell their art online without worrying that their sales, or future trades of their art, encourages or causes damage to the environment. We recommend that artists and others encourage new and existing blockchain platforms, especially Ethereum, to push for the implementation of the ideal solution we propose: Environmentally Smart Contracts for non-fungible tokens.

\section{NON-FunGIBLE TOKENS, BLOCKCHAINS, AND ENVIRONMENTAL IMAPCT}

First suggested in Ethereum Improvement Protocols 721 [5][6], a non-fungible token (NFT) is a small amount of data that certifies something as literally unique. Being literally unique, there is nothing that an NFT could be traded for that is the same in all relevant respects (hence 'non-fungible') [5]. Examples of fungible tokens include amounts of cryptocurrency, such as 1 Bitcoin, which can in theory be perfectly traded for a different 1 Bitcoin. In practice, newly minted Bitcoins may be valued slightly more than old ones [7]. But this is also true for new cash, which may be valued slightly more than grimy, glitter-encrusted old bank notes. And, in both cases, the extra value new versions of the tokens accrue is likely to be negligible.

NFTs exist as an entry on a blockchain - a digital ledger - that all but completely ensures the entry remains discoverable and unaltered for the life of the blockchain. The NFT data includes a record of its creation and every trade it is subject to [8]. By certifying the uniqueness and transaction history of something, NFTs seem well disposed to use for items that gain most or all of their value from their rarity or uniqueness. Prime examples would be collectible items, such as trading cards and many kinds of art [9]. Furthermore, the proof of ownership provided by the blockchain technology underpinning NFTs is especially useful for items that are traditionally very difficult to verify ownership of, such as digital items. For these reasons, NFTs appear to be very useful for securing the value of digital art [5].

Blockchain technology has been widely touted as a force for decentralising and democratising financial [10] and even political [11] institutions. NFTs can also decentralise and democratise art [12]. Many artists around the world struggle because they are not given access to the relevant cultural and economic institutions and establishments required to sell their art profitably. They never get invited to exhibit at the Tate Modern, their art never comes up for auction at Sotheby's, and their local area lacks the market and infrastructure for selling any art. This issue has been compounded for digital artists because of the worries about the authenticity and uniqueness of the art mentioned above [13]. Marketplaces for NFT digital art, such as Opensea, Rarible, and SuperRare, allow any art to be displayed for sale, completely bypassing the gatekeepers of the established 
artworld. All that digital artists must do to be able to securely sell to the world is "mint" their art with NFTs.

The minting process brings an NFT into existence with its first entry on a blockchain digital ledger, thereby securing the value of a digital work of art. But, not much in this world is free. Minting, as with many other actions performed on blockchains, incurs a fee, payable in the native currency of that blockchain. The financial costs of minting vary across platforms and across time as the cost of using blockchains is often dependent on current market forces as well as the rules for how that blockchain and currency operate. In early 2021, prices for minting on platforms using Ethereum were very costly because of high demand [14]. In February, for example, it cost about US\$100 ETH [15] (the native currency of the Ethereum blockchain) to mint a single work. Prices have since settled down considerably, with minting a single work now costing US\$20. But the financial cost isn't the only cost of NFTs.

Many artists considering using NFTs were shocked to discover the considerable environmental costs associated with the major blockchains, such as Bitcoin and Ethereum, and any NFTs that rely on Ethereum [4]. Taking Ethereum as an example, anything done on this blockchain requires paying fees in Ether (ETH). Since Ethereum is a proof-ofwork blockchain, ETH comes into being by a process called "mining". When mining, computers are set to work, competing to be the first to solve a complex mathematical problem. The first miner to solve the problem is rewarded with newly minted cryptocurrency. Successful mining effectively requires dedicated computers and electricity. Lots of electricity. Current estimates suggest that Ethereum miners use the same amount of energy as the country of Greece [16]. Calculating how much damage NFTs do to the environment is very complex [17]. We can estimate how much electricity it takes to mine, say, one ETH, but if we use exactly one ETH to mint and sell several NFTs that ETH doesn't just disappear or get thrown away. It is cycled around to the miners of Ethereum. What the minting does is increase the congestion on the Ethereum blockchain, which pushes up the price of transactions on the blockchain, which increases the attractiveness of mining, meaning more miners use electricity to mine Ethereum. Given that multiple contextual factors, including supply and demand of Ethereum, fossilfuel-based electricity, green electricity, NFTs, and several other goods, affect the amount of electricity used whenever an NFT is minted, measures of environmental impact of minting are rough estimates at best. Nevertheless, the total environmental costs of the whole system are far from negligible and the whole system seems incredibly wasteful. Millions of over-powered computers work away night and day to solve pretend puzzles to provide a level of security for blockchains that could be approximated in other nonpolluting ways.

In proof-of-stake systems, as opposed to the proof-ofwork systems above, the network is secured, and the native cryptocurrency of a blockchain is produced, by people "locking up" that cryptocurrency in the network rather than by energy-intensive mining. Locking up entails individuals depositing the currency into the network as validators [18]. New currency is given randomly to validators based on the extent of their deposits. The more an individual has deposited, the greater chance they have of receiving it. Since proof of stake blockchains don't require computers to do any extra work, this method has something like 99.9\% less environmental impact than the energy-intensive proof of work method currently used by Ethereum [19].

\section{THE DILEMMA FOR ARTISTS}

NFTs provide an exciting opportunity for digital artists to get more exposure to liquid markets, sell more of their work for a higher price, and perhaps make a living. However, many artists see themselves as positive forces in society that spur others into positive states of mind and behaviours [20][21], not as bad examples that encourage waste and environmental degradation. Unfortunately for artists, the current range of options for using NFTs means that they are only likely to get the benefits of higher profits if they mint and sell on Ethereum. There are options to mint and sell NFTs on minor proof-of-stake blockchains, but the audience is much smaller and the sales are much lower. No $\$ 69,000$ pay days appear to be on the horizon in these environmentally friendly blockchains, let alone individual $\$ 69,000,000$ sales. So, the ethical-prudential dilemma for many digital artists is to choose either contributing to the degradation of the environment for higher profits or missing out on the opportunity for higher profits while watching others degrade the environment.

What should a self-respecting, environmentally conscious digital artist do when facing this dilemma? Some artists prioritised prudential concerns, embracing the energyintensive main blockchains. A subset of these attempted to mitigate the environmental damage of their actions by apportioning some of their profits to carbon off-setting. Unfortunately, the majority of carbon-offsetting schemes do not appear to be very helpful, especially in the long-run [4]. Furthermore, paying the schemes in a proof-of-work cryptocurrency or paying to cash out of the cryptocurrency just further add to the environmental costs of the system. More famous artists, such as Beeple [22], can afford to fund carbon-offsetting schemes generously. Up-and-comers, however, are unlikely to be making enough money to undo all or even any of the harm their NFTs cause. And herein lies the main ethical problem with famous artists minting and selling their work on proof-of-work blockchains. Even if they divert a lot of their profits to effective carbon-offsetting schemes, their presence and big sales on a proof-of-work blockchain bring more buyers and more artists to that blockchain, encouraging the growth and entrenchment of an unnecessarily wasteful and polluting system. Non-digital artists that are already famous and successful, and hence don't really need NFTs to achieve a high level of profitability, have even more reason to avoid promoting energy-intensive proof-of-work blockchains by minting NFTs on them. Again, even if they engaged in some carbonoffsetting, it is not clear that they could fully compensate for the harm their potentially talismanic support of this system will inflict on the environment over the years. Lesser-known artists minting NFTs on Ethereum is less consequential, but still morally problematic because their actions also help maintain and grow this system. This moral approbation is possible because these artists all had another option.

A few notable artists and many lesser-known artists minted their NFTs on smaller environmentally unproblematic blockchains, such as Tezos or Phantasma, or on Ethereum sidechains like xDai or Polygon and the newly launched Arbitrum (which uses ETH and has reduced but far from negligible fees) and most interestingly Palm (which 
adds a small carbon offset to transactions). However the biggest marketplaces and the biggest buyers are on Ethereum: 8 of the top 10 NFT marketplaces by sales volume are on Ethereum [23]. Foregoing higher profits for their ethical principles, artists outside the Ethereum chain may be hoping that their ethical principles sustain them through winter. We should commend the principles of artists minting their NFTs on sidechains, but an unfortunate moral hazard lies here too. Even if an artist does sell an NFT on an environmentally friendly side chain such as xDai, it can be "bridged" onto Ethereum. Bridging is the transfer of tokens, including NFTs, from one blockchain to another. Bridging from a side chain to an energy intensive mainchain incurs a mainchain fee (and thereby contributes to environmental degradation). The main reason for bridging NFTs to Ethereum or other mainchains is to increase the options for selling the NFT - as mentioned, the demand and prices are greater on the mainchains than on sidechains like xDai. Owners of NFTs might also bridge them to a larger and more reputable blockchain to increase the security of the proof of uniqueness (the key to NFT's value for digital art). Since many NFT purchases appear to be investments, once they are bridged to a mainchain, they may be traded several times, with each trade contributing more harm to the environment. So, minting and selling on environmentally friendly sidechains like xDai seems like the morally right decision because it avoids the environmental costs of minting on energy-intensive mainchains like Ethereum. However, sidechain minting may still may end up contributing to considerable environmental damage through bridging. All things considered, minting digital art on xDai or other proofof-stake blockchains substantially reduces the prudential benefits of higher profits compared to Ethereum and still contributes to environmental degradation, albeit less so.

Neither of these solutions (mainchaining and carbonoffestting or sidechaining) allows digital artists to take advantage of the opportunities presented by NFTs without supporting an unnecessarily wasteful and environmentally damaging system. So, the dilemma remains.

\section{OUR RECOMMENDATION: ENVIRONMENTALLY SMART CONTRACTS}

The good news is that Ethereum plans to move to a proof-of-stake instead of a proof-of-work system [19]. The bad news is that it might take a while for this to happen. It is planned for this year [19], but it is certainly possible that it takes until 2022 and potentially even later. Artists can help encourage Ethereum to make this change as quickly as possible in several ways. They can refuse to mint or sell their art on any proof-of-work blockchain and let the foundation team at Ethereum know why. They can support the trial and development of Ethereum's proof-of-stake chain, Beacon, including locking in some of the associated cryptocurrency if they can. They can also do what many artists do best - create art that sends a loud message about the environmental ills of proof-of-work blockchains and NFTs associated with them. Of course, they should avoid being hypocritical in the process. Unfortunately, this may rule out minting and selling their art on proof-of-stake side chains because of the bridging problem mentioned above. It seems a more thoroughgoing solution is needed.

In order to combat the likelihood of investors and others bridging NFTs to wasteful and polluting proof-of-work blockchains, Ethereum could harness and enhance the technology of smart contracts to make bridging NFTs automatically internalise the environmental externalities associated with proof-of-work blockchains. The result would be Environmentally Smart Contracts.

Smart contracts, originally proposed by Szabo [24], are enabled by the digital-ledger nature of blockchains. These contracts are smart because they are irreversible, unblockable, and openly trackable. Smart contracts can also be irreversibly encoded with conditions that cannot be removed no matter how many times the NFT the contract is for is traded [5]. Currently, most sites that allow you to mint NFTs also allow you to specify a certain percentage of any sales or resales to go to the original artist in perpetuity [5]. Rare Art Lab is an example [25]. These rules are encoded into the smart contract controlling the NFT and anyone that buys the NFT and then sells it will find that that a percentage is skimmed off the sale price and automatically deposited to the original artist's digital wallet.

Several people have suggested smart contracts use rules like this to apportion some of the sale price of NFTs to go to charities [26][27]. By including contributions to climate focussed not-for-profits (that have proven to be effective; e.g., [28]) in the smart contracts, the tiny environmental costs of minting and selling on a proof-of-stake blockchain can be fully redressed or even overcompensated for. But, dealing with the threat of bridging is slightly more complicated and would require a new kind of smart contract.

Smart contracts could be coded to either prevent, or add conditions to, bridging the associated NFT to a proof-ofwork blockchain. In light of the prudential concerns of artists (and others) and the decentralising and democratising affordances of blockchains, we recommend including conditions on bridging to proof-of-work blockchains rather than a prohibition. An Environmentally Smart Contract (ESC) would be coded to add a large environmental tax to any sale or movement of the associated NFT on any proofof-work blockchain. The tax needs to be large enough to be sure that the environmental costs are completely redressed and that an additional "punitive damages" amount is added to the tax to further dissuade use of wasteful and polluting blockchains to mitigate any environmental costs that occur via general increased interest in the blockchain generated by trades on it. Directly linking the tax to the transaction fee at the time of sale or movement seems the best way to achieve this.

The advantages of ESC, as defined here, should be clear. Most importantly, the environmental taxes mean that trades of the associated NFT are all but guaranteed to be net positive for the environment. Also very important, the increased environmental tax for bridging and any future sales on proof-of-work blockchains dissuades individuals from bridging NFTs away from proof-of-stake blockchains, while also dissuading buyers and sellers on proof-of-work blockchains (who will likely share the cost of the environmental tax). This means that as long as artists use ESCs, they can mint and trade as they please, with little risk of losing out on higher profits, or of incurring the moral hazard of contributing to environmental degradation. So, digital artists, especially, should do what they can to petition Ethereum and other blockchains to facilitate the creation of Environmentally Smart Contracts. Perhaps they can even use their artistic skills in this endeavour. 


\section{REFERENCES}

[1] M. O'Brien and K. Chan, "How two friends made art history buying a US\$70M digital work," CTV News, 26 march 2021. https://www.ctvnews.ca/sci-tech/how-two-friends-made-art-historybuying-a-us-70m-digital-work-1.5363510/nft-1.5363520

[2] G. Kay, "We talked to crypto-art investors to figure out what's driving people to spend millions on NFTs, despite no guarantee their value will increase," Business Insider Australai, 20 March 2021. https://www.businessinsider.com.au/why-are-people-buying-nftsinvesting-in-nft-crypto-art-2021-3? $\mathrm{r}=\mathrm{US} \& \mathrm{IR}=\mathrm{T}$

[3] T. Hasleton, "How to make, buy and sell NFTs," CNBC, 23 March 2021. https://www.cnbc.com/2021/03/23/how-to-create-buy-sellnfts.html

[4] V. Di Liscia, "As NFT Sells for \$69M, Artists question environmental impact of blockchain,” Hyperallergic, 11 March 2021. https:/hyperallergic.com/628845/as-nft-sells-for-69m-artistsquestion-environmental-impact-of-blockchain/.

[5] Q. Wang, R. Li, Q. Wang, and S. Chen, "Non-fungible token (NFT): overview, evaluation, opportunities and challenges," arXiv preprint arXiv:2105.07447, 16 May 2021.

[6] E. William, S. Dieter, E. Jacob, S. Nastassia, "Erc-721 non-fungible token standard. Ethereum improvement protocol, EIP-721," Ethereum, 2018. https://eips.ethereum.org/EIPS/eip-721

[7] H. Korin, “Are Your Bitcoins Clean or Dirty?” ORBS, July 2021. https://www.orbs.com/are-your-bitcoins-clean-or-dirty/

[8] M. Franceschet, G. Colavizza, T. Smith, B. Finucane, M. L. Ostachowski... "Crypto art: A decentralized view," Leonardo, 2020, pp. 1-8.

[9] J. Fairfield and L. J. Trautman, "Virtual art and non-fungible tokens," unpublished, SSRN, $11 \quad$ April 2021. https://ssrn.com/abstract=3814087

[10] S. Abdulhakeem and Q. Hu, "Powered by blockchain technology, DeFi (decentralized finance) strives to increase financial inclusion of the unbanked by reshaping the world financial system," Modern Economy, 2021, 12, pp. 1-16.

[11] M. Atzori, "Blockchain technology and decentralized governance: Is the state still necessary?" Journal of Governance and Regulation, 2017, 6(1), pp. 45-62.

[12] I. Pandey, "NFTs are democratizing the art industry for everyone," Hackernoon, 17 June 2021. https://hackernoon.com/nfts-aredemocratizing-the-art-industry-for-everyone-nfp35el
[13] L. Johnston, "The problem with art on digital platforms is..." The Space, no date. https://www.thespace.org/resource/problem-artdigital-platforms

[14] C. Harper and C. Kim, "Ethereum gas limit hits $15 \mathrm{M}$ as ETH price soars," Coindesk, 23 April 2021. https://www.coindesk.com/ethereum-gas-limit-eth-price-soars

[15] Shashwat, "How much does it cost to mint an NFT?," Nerds Chalk, 15 March 2021. https://nerdschalk.com/nft-minting-cost/

[16] Digiconomist, "Ethereum energy consumption index," no date. https://digiconomist.net/ethereum-energy-consumption/

[17] K. McDonald, "Computing per-transaction responsibility for ethereum," GitHub, $19 \quad$ April 2021. https://github.com/kylemcdonald/ethereum-nftactivity/blob/main/Per-Transaction\%20Models.ipynb

[18] O. Ogino, "Proof-of-stake (POS)," Ethereum, 16 April 2021. https:/ethereum.org/en/developers/docs/consensus-mechanisms/pos

[19] C. Beekhuizen, "A country's worth of power, no more!" Ethereum, 18 May 2021. https://blog.ethereum.org/2021/05/18/country-power-nomore/

[20] Artwork Archive, "What is the artist's role in society?" no date. https://www.artworkarchive.com/blog/what-is-the-artist-s-role-insociety

[21] O. Eliasson, "Why art has the power to change the world," World Economic $\quad$ Forum, $18 \quad$ January 2016. https:/www.weforum.org/agenda/2016/01/why-art-has-the-power-tochange-the-world/

[22] J. Kastrenakes, "Beeple sold an NFT for \$69 million," The Verge, 11 March 2021. https://www.theverge.com/2021/3/11/22325054/beeplechristies-nft-sale-cost-everydays-69-million

[23] DappRadar, "NFT Marketplaces," no date. https://appradar.com/nft/marketplaces

[24] N. Szabo, "Smart contracts: building blocks for digital markets," Extropy: The Journal of Transhumanist Thought, 1996, 8(1), 50-53.

[25] R.a.r.e art lab. https://www.lagelnd.com/rare

[26] Pixelplex, "Blockchain charity projects and their philanthropic benefits," no date. https://pixelplex.io/blog/blockchain-charityprojects-and-their-philanthropic-benefits/

[27] B. Hu and H. Li, "Research on charity system based on blockchain," 2020 IOP Conf. Ser.: Mater. Sci. Eng. 768072020

[28] M. Gunther, "Which are the most effective climate change nonprofits?" Nonprofit Chronicles, 12 December 2020. https://medium.com/nonprofit-chronicles/which-are-the-mosteffective-climate-change-nonprofits-d1083f0a $2 \mathrm{f0} 2$ 